\title{
C-SELECTION AND S-SELECTION IN INHERITANCE PHENOMENA
}

\author{
TAKANE ITO \\ University of Tokyo
}

\begin{abstract}
In this paper, we argue that inheritance of c-selection and inheritance of s-selection require mutually independent description. Support for this argument is found in several cases where only one or the other of the two types of selection is inherited. These examples, which may appear totally irregular, prove to be semiregular cases that can be given a reasonable account only by assuming that the two types of inheritance are independent of each other. This argument leads us to a view of the lexicon where syntactic information and semantic information are mutually independent.*
\end{abstract}

0. InTRODUCTION. It is commonly observed that morphologically related words share selectional restrictions and take arguments/complements of the same kind.

(1) a. John departed from London.

b. John's departure from London

In unmarked cases like 1 , this phenomenon, which is called inheritance in the morphological literature, can be captured in terms of either categorial selection (c-selection) or semantic selection (s-selection): departure can be considered to inherit from its source verb depart the c-selectional requirement that it take a PP complement, or the s-selectional requirement that it take Source. ${ }^{1}$ Then, a question arises: Can we do only with

* This is a revised version of the paper I presented at the 8th National Conference of the English Linguistic Society of Japan on November 17, 1990. Earlier versions were also read at meetings of Tokyo University English Linguistic Circle and Morphology Study Group. I am grateful to the audience of these presentations for valuable comments, and to $\mathrm{K}$. Hasegawa, T. Takahashi and two EL reviewers for their comments and criticisms on an earlier draft. I am also indebted to Brent de Chene and Adam Kabat for their help in constructing English data.

${ }^{1}$ It seems likely that some more elaborated device such as Jackendoff's 1990 conceptual structure is required to represent s-selectional properties, but we employ familiar $\theta$-role names, simply for expository purposes. We may readily reinterprete 'Goal', for instance, as 'the argument of the Path-function TO' (Jackendoff 1990: 47), if

English Linguistics 8 (1991) 52-67 - 52-

(C) 1991 by the English Linguistic Society of Japan 
one or the other of the two types of selection?

This paper will argue that, in spite of apparent redundancy, inheritance must be captured both as the inheritance of c-selection (c-inheritance) and as the inheritance of s-selection (s-inheritance), and that the syntactic aspect and the semantic aspect must be captured independently of each other. By separating c- and s-inheritance, we can give a natural account of seemingly exceptional or irregular cases.

1. C-INHERITANCE AND S-INHERITANCE. It has sometimes been argued that it is not appropriate to describe inheritance in terms of c-selection (Booij 1988, Hoekstra \& Putten 1988). If we take the description of s-inheritance to be necessary, the description of c-inheritance might appear redundant, given a reasonable assumption that each argument type has its typically associated form(s) of syntactic realization, which is called canonical structural realization (CSR) (Grimshaw 1981: 174, Chomsky 1986: 87). In 1, for instance, the noun departure s-inherits Source from depart. Then, given that the CSR of Source is a PP, we no longer need to say it c-inherits a PP. If the association between argument types and their CSR were without exception, it would be totally redundant to describe c-inheritance in addition to s-inheritance. But CSR, as is implicit in the word 'canonical', is not an absolute rule, and in exactly those cases which show some irregularities concerning CSR, we find the description of c-inheritance non-redundant.

(2) a. His parents tend toward strictness.

b. His parents tend to be strict.

(3) a. the tendency of his parents toward strictness

b. the tendency of his parents to be strict

(4) a. His parents lean toward strictness.

b. *His parents lean to be strict.

(5) a. the leaning of his parents toward strictness

b. *the leaning of his parents to be strict

In the above examples, the verbs tend and lean are nearly synonymous, and both take two arguments, Theme and Goal. ${ }^{2}$ Examples in 2 show

it is necessary. A list of $\theta$-role names may appear to be a syntactic entity like argument structure of Grimshaw 1990. But we take s-selection to be semantic in nature, in that it represents lexical-semantic properties of a given item. We will turn to this point in $\$ 3$. 
that the CSR of the Goal argument is either a PP or an infinitival clause. This is supported by the syntactic behavior of some other synonymous expressions like incline, be disposed, and be prone, which cooccur with either a PP or an infinitival clause. As shown in 4, however, the verb lean may take a PP but not an infinitival clause. Thus, the two verbs in question differ in c-selection, though they share s-selection. Importantly, the difference in c-selection is inherited by their derived nouns tendency and leaning, as shown in 3 and 5 . This cannot be given a reasonable account unless we have some mechanism to deal with c-inheritance. As for s-inheritance, both tendency and leaning inherit Goal. If we were to assume that c-selectional information is not inherited, we would wrongly predict, given the CSR of the Goal argument, that both tendency and leaning may cooccur with either a PP or an infinitival clause. In other words, the description of c-inheritance is essential to capture the correlation between the unacceptability of $4 \mathrm{~b}$ and that of $5 \mathrm{~b}$.

Examples like the following also support the above argument:

(6) a. The lawyer advised him to drop the case.

b. The lawyer advised him that he drop the case.

(7) a. the lawyer's advice to drop the case

b. the lawyer's advice that he drop the case

(8) a. *The lawyer suggested to him to drop the case.

b. The lawyer suggested to him that he drop the case.

(9) a. *the lawyer's suggestion to drop the case

b. the lawyer's suggestion that he drop the case

Though the verbs advise and suggest are near synonyms and s-select the same types of arguments (Agent, Goal and Theme), their c-selection is different as to whether they allow infinitival complements, ${ }^{3}$ and the c-

2 The term Goal might be inappropriate here, but the exact naming of argument types is irrelevant to our discussion. What is important is that the two verbs in question are nearly synonymous so that they can reasonably be assumed to take the same type of argument, whatever the appropriate name is. See fn. 1 above.

3 The ungrammaticality of $8 \mathrm{a}$ is not due to the fact that the Goal argument (to him) is realized with a preposition $t o$, for we find acceptable examples of the same syntactic construction:

(a) He shouted to her to go away.

(b) The policeman signaled to us to stop.

Hence, the ill-formedness of $12 \mathrm{a}$ cannot be attributed to control theoretic or some other syntactic/semantic deviance, and must be given a lexical treatment as a cselectional violation. 
selectional discrepancy is inherited by the derived nouns.

As for s-inheritance, its necessity seems fairly obvious. Consider the following examples:

(10) a. They donated $\$ 1,000$ to an orphanage.

b. *They donated $\$ 1,000$ from an orphanage.

(11) a. their donation of $\$ 1,000$ to an orphanage

b. *their donation of $\$ 1,000$ from an orphanage

The verb donate c-selects an NP and a PP, and s-selects Agent, Theme and Goal. 10b is a violation of s-selectional restriction, for from $+\mathrm{NP}$ can never be interpreted as Goal. The ill-formedness of $11 \mathrm{~b}$ is evidently related to that of $10 \mathrm{~b}$. If we were concerned only with c-inheritance and ignored s-inheritance, the noun donation would inherit a PP from donate, but no semantic information would be inherited as to the types of the arguments it takes. Then the inherited PP could be Source, or, for that matter, anything whose CSR is a PP. Thus without s-inheritance, the parallelism between $10 \mathrm{~b}$ and $11 \mathrm{~b}$ could never be captured. Similar examples to support the point is quite easy to construct, and I believe this brief discussion is enough to show the necessity of s-inheritance.

Then, our conclusion is that a plausible theory of inheritance requires the description of both c- and s-inheritance.

2. MUTUAL INDEPENDENCE OF C- AND S-INHERITANCE. In the examples so far discussed, both c- and s-selection are inherited, so that the description of the two types of inheritance appears somewhat redundant. But in this section, we will show that there are some cases where only one or the other of the two types of inheritance is observed. $\$ 2.1$ deals with cases where only s-selection is inherited. $\$ 2.2$ is concerned with cases where only c-inheritance is observed. Consideration of these cases leads us to conclude that the two types of inheritance are independent of each other, and hence the description of the two is not always redundant.

\subsection{S-INHERITANCE ONLY.}

2.1.1. Deverbal nOUNS AND ADJECTIVES. Nouns and adjectives do not take bare NP complements even if they are derived from transitive verbs. As is well-known, in many cases the argument corresponding to the verb's NP complement appears as of+NP in a nominal/adjectival construction:

(12) a. His silence indicates disapproval.

b. the indication of disapproval 
c. His silence was indicative of disapproval.

Since Chomsky 1970, it has often been argued that of $+\mathrm{NP}$ in $12 \mathrm{~b}$, c is not a PP but an NP at D-structure, and of is automatically inserted at some later level. If this argument is tenable, examples like 12 show full regularity concerning inheritance: the deverbal noun/adjective inherits an NP complement from the base verb, and of is inserted afterwards.

However, we also find many cases where prepositions other than of appear with the argument corresponding to the NP complement of the base verb. In the following examples, ${ }^{4}$ a deverbal noun takes $t o+\mathrm{NP}$. The base verb cannot cooccur with $t o+\mathrm{NP}$, and hence we cannot take the $\mathrm{PP}$ complement to+NP in $13 \mathrm{~b}$ to be inherited from the base verb. The same holds in every set of examples in this subsection.

(13) a. He resembles (*to) his father.

b. his resemblance to his father

approach/approach, contradict/contradiction, obstruct/obstruction, hinder/hindrance, obey/ obedience, disobey/disobedience, offend/offense

Some deverbal adjectives such as destructive, obedient, offensive, interesting, and attractive also take to+NP though their base verbs do not.

Prepositions other than to are also observed: we find from in 14, for in 15 , on in 16, against in 17-18, at in 19, into in 20 , between in 21 , with in 22 , about in 23 and over in 24.

(14) a. They robbed the woman of the jewels.

b. *They robbed (of) the jewels from the woman.

c. the robbery of the jewels from the woman

(15) a. They respect (*for) the priest.

b. the respect for the priest admire/admiration, esteem/esteem, scorn/scorn, desire/desire

(16) a. The enemy attacked (*on) the city.

b. the attack on the city assault/assault, discuss/discussion, research/research

\footnotetext{
${ }^{4}$ In this section, under each set of examples, I will cite base/derivative pairs that show the same syntactic distribution as the set of examples, if I know of some. I simply give a list of base/derivative pairs which take the same preposition. So the examples given below each set may or may not share the type of argument they take. In 16, for instance, the on-phrase that occurs with attack or assault is likely to be of different argument type from the on-phrase with discussion or research.
} 
(17) a. They accused the woman of theft.

b. *They accused (of) theft against the woman.

c. the accusation of theft against the woman suspect/suspicion

(18) a. They attacked (*against) the city.

b. their attack against the city assault/assault, resent/resentment

(19) a. He attempted (*at) constructing a flying machine.

b. his attempt at constructing a flying machine regret/regret, resent/resentment

(20) a. The teacher entered (*into) the room.

b. the teacher's entrance into the room investigate/investigation

(21) a. It is often useful to compare (*between) two things.

b. It is useful to make a comparison between two things. contrast/contrast, confuse/confusion, divorce/divorce, connect/connection

(22) a. He indulged (*with) his children in material posessions.

b. He was indulgent with his children.

(23) a. They discussed (*about) the problem.

b. the discussion about the problem

(24) a. You have to control (*over) your feelings.

b. You have to have good control over your feelings.

In these examples, s-selection is evidently inherited, and only c-inheritance is blocked. ${ }^{5}$ Thus we take them to be semiregular cases, where only one of the two types of inheritance is observed.

The same situation obtains in other languages as well. For example, Sugioka (1989: 184, fn. 2) points out similar examples in Japanese, and Hoekstra \& Putten (1988: 182f) and Booij (1988: 61) refer to similar examples in Dutch.

Consideration of such cases as these has led some linguists including Booij 1988 and Hoekstra \& Putten 1988 to argue that the description of c-

\footnotetext{
5 Many of the examples in 13-24 are so-called result nominals. It is often pointed out that result nominals show less regularity as to inheritance than action nominals, and some argue that they do not take part in inheritance phenomena at all. See Shimamura (1990: 98-104) and the references cited there for detailed discussions. But it appears evident that s-inheritance is observed in 13-24. It might be the case that result nominals tend to block c-inheritance more readily than action nominals.
} 
inheritance is inappropriate to capture the regularity concerning inheritance phenomena. In these examples, c-selection of the base verb is not inherited by the derivative, and the observed regularity is statable only as s-inheritance, and hence inheritance cannot be captured in terms of cselection. I agree that in these cases c-inheritance is not observed. As we saw in the preceding section, however, there are inheritance phenomena which require c-selectional description. So, the cases in question lead us to deny c-inheritance only in these particular cases, not in general.

Of course, it is not a mere accident that these derived nouns/adjectives do not inherit NP complements. Nouns and adjectives in general cannot take bare NP complements, and this should be given a principled syntactic explanation, presumably on the basis of case theory. Then, it need not be stipulated as a property of each derived word or word formation (WF) process. Assuming that the CSR of the relevant argument types is either an NP or a PP, it is fully predictable that the derived nouns/adjectives take PP's. In other words, the examples under consideration are quite regular in a sense, and we may well call these cases 'principled exceptions'.

Let us make a digression on some different approaches, for, given this regularity, one might very well argue that there is no blocking of inheritance in these examples. One possibility is to claim that the apparent PP's in these examples are NP's at the level of D-structure. There seem to be two possible approaches along this line. One is to generalize the rule of of-insertion to other prepositions. The other is to assume that the prepositions in these examples do not head PP's, but are superficial realization of abstract cases the following NP's bear: of is an accusative marker, to is a dative marker, and so on. But neither approach seems tenable, given the wide variety of prepositions that appear in examples like 13-24. In the former approach, the insertion rule must insert an appropriate preposition in each case, which certainly must depend on the meaning of the preposition. But such automatic insertion of semantically loaded words seems undesirable. In the latter approach, we would have to claim that there are as many abstract cases as there are prepositions, which is highly implausible. Thus, it is not an appealing claim that the PP's in these examples are actually NP's at D-structure.

Another possible approach is to claim that verbs as well as derived nouns/adjectives c-select PP's, and the prepositions are incorporated into 
the verbs at some prelexical level, as in Gruber 1976. ${ }^{6}$ This approach requires some lexical feature, call it $[ \pm \mathrm{I}]$, to mark the possibility of preposition incorporation. A verb like those in 13-24, which cannot cooccur with an overt preposition, is marked [+I], a verb which cannot incorporate the preposition like arrive at is marked [-I], and a verb which optionally incorporates the preposition like meet with (cf., meet (with) a person) is marked $[ \pm \mathrm{I}]$. Obviously, this feature plays the same role as our c-selection, in that it guarantees in what syntactic context a given verb occurs. The c-selectional feature $[+\mathrm{I}]$ is not inherited in the cases under consideration, and this should be treated by some general principle which confirms that the feature [+I] is never compatible with nouns/adjectives. Then, the derived nouns/adjectives may be considered to inherit PP complements from their ancestors, but they do not inherit the c-selectional feature $[+\mathrm{I}]$. This seems to be equivalent to our claim that deverbal nouns/adjectives are principled exceptions.

Thus we may safely conclude that c-selection is not inherited in 13-24, and that these are semiregular in that they inherit only one of the two types of selectional restrictions.

2.1.2. UnPREDICTABLE CASES. We also find some cases where cinheritance is blocked without any apparent principled reason. First, consider the following examples:

(25) a. He discovered that the earth is round.

b. *the discoverer that the earth is round

c. the discoverer of the fact that the earth is round As is often pointed out (Hoekstra \& Putten 1988: 169f, Grimshaw 1990: 101), agentive nouns with the suffix -er do not inherit tensed clauses. $25 \mathrm{c}$ shows that propositional Theme is regularly inherited, and so it must be c-inheritance that is blocked here. Then, we have to stipulate that -er suffixation blocks the c-inheritance of a tensed clause. (But see Grimshaw (1990: 101-04) for an attempt to systematically exclude sentential complements from -er nominals.)

25 is a case that can be captured as an idiosyncracy of a particular affix. We also find blocking of c-inheritance that must be specified as a property of each derived word, rather than of any particular affix or WF

${ }^{6}$ I am grateful to T. Kageyama, who pointed out this possibility to me in the question/answer period of my presentation at the ELSJ conference. 
process:

(26) a. We hope to win the race.

b. *our hope to win the race

c. our hope of winning the race

(27) a. It is important for John to arrive on time.

It is important that John arrive on time.

b. *the importance for John to arrive on time *the importance that John arrive on time

c. the importance of John's arriving on time odd/oddness, curious/curiousness

In these examples, clausal complements are not inherited. The acceptability of the c-examples shows that s-selection is regularly inherited. Deverbal/deadjectival nouns do not always show this type of exceptional behavior:

(28) a. our desire to win the race

b. the necessity for John to arrive on time

c. the necessity that John arrive on time

Thus the blocking of c-inheritance in 26-27 should be captured as an idiosyncratic property of each lexical item.

Here, again, we may safely claim that examples like 25-27 constitute semiregular cases: regular in that they inherit s-selection, but irregular in not inheriting c-selection.

2.2. C-Selection Only. Now let us turn to examples that inherit cselection but not s-selection. First, consider the following examples: ${ }^{7}$

(29) a. connect the hose to the faucet

b. * connect the hose from the faucet

c. *disconnect the hose to the faucet

7 The distribution of connect/disconnect is pointed out in Namiki (1985: 150). We may add to our list the following examples pointed out also in Namiki (1985: 150):

(a) arm him with $/ *$ of a gun

(b) disarm him *with/of a gun burden/disburden, charge/discharge

We do not have space enough to discuss how these should be analyzed in $\theta$-theoretic terms, and here I simply note that these are likely to give another supporting evidence to our argument.

It should be pointed out that $u n$ - in $29-31$ is a reversative prefix attached to verbs, which must be distinguished from a negative prefix $u n$ - attached to adjectives. (Cf. Siegel 1973: 302, fn. 3) 
d. disconnect the hose from the faucet

hitch/unhitch, tie/untie, leash/unleash, link/unlink, couple/uncouple, glue/unglue

(30) a. engage oneself in a project

b. *engage oneself from a project

c. *disengage oneself in a project

d. disengage oneself from a project entangle/disentangle, place/displace, load/unload

(31) a. wrap a scarf around one's neck

b. *wrap a scarf from around one's neck

c. *unwrap a scarf around one's neck

d. unwrap a scarf from around one's neck

Though different prepositions appear in the a-examples, the situation seems essentially the same. The base verb c-selects an NP and a PP, and $\mathrm{s}$-selects Theme and Goal. The c-selection is shared by the derived verb, but the s-selection is not: the derived verb does not inherit Goal and takes Source instead.

It might appear plausible to claim, as in Smith 1981, that the prefix disor $u n$-itself bears the meaning of 'separation' and takes Source. But we can hardly claim that verbs with dis- or un- always take Source or from + NP:

$$
\begin{aligned}
& \text { a. *disapprove NP from NP, *discredit NP from NP, } \\
& * \text { dishonor NP from NP, *disobey NP from NP } \\
& \text { b. *unfreeze NP from NP, *unlearn NP from NP, } \\
& \text { *unlock NP from NP, *unsettle NP from NP }
\end{aligned}
$$

Roeper (1981: 149, fn. 3), noting examples like 32a, claims that the phenomenon exemplified in 29-31 does not generalize. But he fails to note the clear difference between the verbs in 29-31 and those in 32. Unlike the verbs in 29-31, none of the verbs in 32 nor their base verbs c-select PP's. Given this clear contrast, a very plausible analysis suggests itself: when a reversative prefix dis- or un-attaches to a verb that c-selects a PP and s-selects Goal, the derived verb c-inherits the PP, but the s-inheritance of Goal is blocked by virtue of the semantic change caused by the prefixation, and the derived verb s-selects Source, which is semantically opposite to Goal. This change in s-selection is observed only when the derived verb inherits from the base the c-selectional requirement to take a PP: the shared PP complement alternates between Goal and Source as in 29-31. This natural account is possible only in a framework which states c-inheritance and s-inheritance separately, where these examples 
can be treated as semiregular cases of inheriting c-selection without inheriting s-selection.

Next, let us turn to the following examples: ${ }^{8}$

(33) a. It has not yet been determined that she has escaped.

b. It has not yet been determined whether she has escaped.

c. *It is still undetermined that she has escaped.

d. It is still undetermined whether she has escaped.

(34) a. He has not yet decided to go.

b. He has not yet decided where to go.

c. ${ }^{*} \mathrm{He}$ is undecided to go.

d. He is undecided where to go.

As is convincingly argued in Ota \& Kajita (1974: 397-403) and Grimshaw 1979 , it is a matter of s-selection, not of c-selection, whether a lexical item takes a that clause or a $w h$-clause. The same is true of the choice between an infinitival clause with no complementizer ( $\phi$-infinitive) and a $w h$-infinitive. The that clause in 33a and the $\phi$-infinitive in $34 \mathrm{a}$ are realization of Proposition arguments, while the $w h$-clause in $33 \mathrm{~b}$ and the $w h$ infinitive in $34 \mathrm{~b}$ are realization of Question arguments. Thus, the verbs in 33-34 can be analyzed as follows: determine c-selects a tensed clause, while decide c-selects an infinitival clause, and both s-select either Proposition or Question. As shown in (33-34)c, the derived adjectives with the negative prefix un- do not inherit every complement/argument the base verbs take. But if we conclude from this that inheritance is not observed in these examples, the parallelism between (33-34)d and (33-34)b would remain uncaptured. It is most plausible to analyze these as a semiregular case of not inheriting a part of s-selection. They regularly cinherit a clausal/infinitival complement, but they inherit s-selection only in part: they inherit Question, but not Proposition.

The blocking of inheritance of Proposition is certainly related to the negativity of the prefix un-, but adjectives with un- do not always reject Proposition:

(35) a. It is unquestionable that he is guilty.

b. $\mathrm{He}$ is unwilling to go.

Therefore, the unacceptability of (33-34)c cannot be attributed only to the prefix $u n-$; it should be considered as a result of the interaction between the s-selection of the base and the semantic change caused by the

${ }^{8}$ Examples in 33 are cited from Bresnan (1972: 71). 
prefixation of un-.

No matter how the interaction in question is to be captured, it is important to us that, if we did not separate c- and s-inheritance, we could not give an adequate description to the fact that $\mathrm{c}$-selectional restrictions are regularly inherited in 33-34.

2.3. Mutual INDEPENDENCE OF SYNTACTIC AND SEMANTIC INFORMATION IN THE LEXICON. So far in this section, we have seen several semiregular cases: in the examples in $\$ 2.1$, only s-selection is regularly inherited, while in the examples in $\$ 2.2$, c-selection but not s-selection is inherited. It is significant to take into account the type of the WF process involved in each set of examples. In \$2.1.1, we dealt with deverbal noun/ adjective formation, and in $\$ 2.1 .2$, we took up the -er agent noun formation, deverbal noun formation, and deadjectival noun formation. All these processes change the syntactic category of the base. On the other hand, the WF processes involved in the examples discussed in $\$ 2.2$ are prefixation of reversative dis-, reversative un-, and negative $u n-$, which all affect the meaning, rather than the syntactic category, of the base. This cannot be a mere coincidence, and we are led to a very natural hypothesis that category-changing WF processes may block c-inheritance (i.e. affect c-selection), while meaning-changing WF processes may block s-inheritance (i.e. affect s-selection). In other words, there is a reasonable correlation between the type of a WF process and the effect it may have on lexical information: WF processes that affect the syntax of the base may cause a change in the syntactic part of lexical information, while those which affect the semantics of the base may cause a change in the semantic part of lexical information.

In this context, it is worth noting that it is not always obvious whether the observed blocking of inheritance is syntactic or semantic in nature. For instance, let us consider the following examples: ${ }^{9}$

(36) a. *We disbelieve that he is honest.

b. *We disagreed that we should start early.

c. *We disagreed to start early.

Verbs with the prefix dis- do not always reject sentential complements (They can neither prove nor disprove that it is genuine), but it seems true that the reversative prefix tends to block the inheritance of clauses. If it

${ }^{9}$ I am grateful to an $E L$ reviewer for bringing examples like 36 to my attention. 
is the c-inheritance of clausal complements that is blocked here, these examples constitute counterexamples to our hypothesis, for dis- is evidently not category-changing. A careful study of these verbs suggests, however, that it is the s-inheritance of propositional arguments that is blocked. The following examples show that disbelieve, unlike believe, cannot take propositional Theme even if it is realized as an NP:

(37) a. I'm told that he's been in prison, but I can't believe it. b. *I'm told that he's been in prison, but I disbelieve it.

The same argument holds in the following:

(38) a. We agreed on starting early.

b. *We disagreed on starting early.

The verb disagree, unlike agree, cannot take as its argument the propositional content of disagreement even if it is realized as on+NP; only the topic of disagreement seems to be allowed to occur as on+NP (We disagreed on the time of departure). Thus we may safely conclude that examples like 36 are not counterexamples to our hypothesis.

Of course, we need more detailed studies of a wider range of WF processes and inheritance phenomena before drawing a conclusion as to the validity of our hypothesis. And yet, this hypothesis seems quite natural and promising in that it provides us with a plausible form of mutual independence of syntactic information and semantic information in the lexicon. And I would like to emphasize again that such a hypothesis is made possible only by assuming that c-inheritance and s-inheritance are described independently of each other.

3. ON LEXICAL REPRESENTATIONS. Before concluding our discussion, let us consider what implications our arguments have on the theory of lexical representations. I believe it is reasonable to assume that inheritance phenomena reflect a speaker's knowledge of lexical items in a fairly straightforward way, especially in productive WF processes. Then, a careful study of inheritance phenomena is likely to provide us with a promising way to gain insight into the theory of lexical representations.

First, our argument in $\S 1$ shows that the information on c-selectional restrictions occupies an essential part in lexical entries. It has sometimes been argued (Pesetsky 1982: 180-205, Chomsky 1986: 86-90) that, given sselection, CSR and case theory, c-selection can be eliminated from lexical entries. But the inheritance phenomena exemplified in 2-9 cannot be adequately described if lexical entries do not include c-selectional information. Specifically, these examples show the necessity to refer to the 
distinction between a PP and a clause, or between a tensed clause and an infinitival clause, where case theory does not help. Hence, we may safely conclude that c-selection is indispensable.

Our second point is concerned with s-selection. As I mentioned in fn.1, the s-selection that is involved in inheritance phenomena is semantic in nature, in that it must refer to semantic properties of arguments. In the case of examples 10-11, for instance, the distinction between Source and Goal is crucial. In this respect, our s-selection is different from Grimshaw's 1990 argument structure (AS), Rappaport \& Levin's 1988 (R\&L) predicate argument structure (PAS), or Zubizarreta's 1987 lexico-semantic structure (S-R), which contains information on the number but not the semantic types of arguments. AS, PAS and S-R, which are devoid of semantic content, do not serve to capture s-inheritance. As for lexical conceptual structure (LCS) of Grimshaw, R\&L, or Jackendoff 1990, I am not sure whether we can identify our s-selection with this representation. It seems reasonable to assume that LCS, a lexical semantic representation, includes information on non-argument adjuncts and implicit arguments, but we have not taken into account how these entities are involved in inheritance phenomena. Thus we have to leave this open for further study.

Our claim that both c-selection and s-selection are necessary for lexical representations may raise a question: Is AS or PAS necessary as an independent lexical representation? There has been a controversy over this issue: Jackendoff (1990: §2.2) argues that (P)AS is simply an abbreviation of a part of LCS, denying its independent status, while R\&L and Grimshaw take (P)AS as an independent lexical representation that mediates LCS and D-structure. This question is far beyond the scope of the present study, but, as far as our data are concerned, we may claim that inheritance phenomena do not motivate (P)AS as a representation independent of c- and s-selection.

Lastly, given our arguments in $\S 2$ that c- and s-inheritance are independent of each other, it is reasonable to assume that c-selection and s-selection are specified independently of each other. Thus we may claim that our discussions give support to Grimshaw's 1979 convincing arguments for the mutual independence of c-and s-selection. Then, we should not try to incorporate both c-selectional information and s-selectional information into one representation. For instance, such lexical representations as elaborated in Woolford 1984 or in Levin \& Rappaport 1986, where the information as to whether a given argument is syntactically realized 
as a PP or an NP is incorporated into s-selectional representation, cannot deal with the data discussed in $\S 2$.

4. Conclusion. We have argued that we need to describe both c-inheritance and s-inheritance, and that the two should be treated independently of each other. This suggests that c-selection and s-selection are both required as lexical information, and that they are specified independently of each other. Our lexicon, as it stands, certainly contains much redundant information, especially with respect to unmarked cases like 1 . Then, it is significant to attempt to reduce such redundancy, but such an attempt should not be directed toward eliminating c-selection or c-inheritance altogether from the theory of the lexicon.

\section{REFERENCES}

BAKER, C. L., and JOHN J. MCCARTHY. (eds.) 1981. The logical problem of language acquisition. Camridge, MA: MIT Press.

BOOIJ, GEERT. 1988. The relation between inheritance and argument linking: Deverbal nouns in Dutch. In Everaert et al., 57-73.

BRESNAN, JOAN. 1972. Theory of complementation in English syntax. MIT dissertation. [Published, New York: Garland, 1979]

CHOMSKY, NoAM. 1970. Remarks on nominalization. Readings in transformational grammar, ed. by Roderick A. Jacobs and Peter S. Rosenbaum, 184-221. Waltham, MA: Ginn and Company.

- 1986. Knowledge of language: Its nature, origin, and use. (Convergence series.) New York: Praeger.

EVERAERT, MarTin; ARNOLD Evers; RINY HuYbregts; and MieKe TromMELEN. (eds.) 1988. Morphology and modularity: In honor of Henk Schultnik. (Publications in languages sciences, 29.) Dordrecht: Foris.

GRIMSHAW, JANE. 1979. Complement selection and the lexicon. LI 10.279-326.

- 1981. Form, function, and language acquisition device. In Baker \& McCarthy, 165-82.

- 1990. Argument structure. (Linguistic Inquiry monographs, 18.) Cambridge, MA: MIT Press.

GRUBER, JEFFREY S. 1976. Lexical structures in syntax and semantics. Amsterdam: North Holland Publishing Company.

Hoekstra, Teun, and FRANS VAN DER PUTTEN. 1988. Inheritance phenomena. In Everaert et al., 163-86.

JACKENDOFF, RAY. 1990. Semantic structures. (Current studies in linguistics, 18.) Cambridge, MA: MIT Press.

LEVIN, BETH, and MALKA RAPPAPORT. 1986. The formation of adjectival passives. LI 17.623-61. 
NAMIKI, TAKAYASU. 1985. Gokeisei. (Shin eibunpo sensho 2.) Tokyo: Taishukan. OTA, AKIRA, and MASARU KAJITA. 1974. Bunpo-ron II. (Eigogaku taikei, 4.) Tokyo: Taishukan.

PESETSKY, DAVID MICHAEL. 1982. Path and categories. MIT dissertation.

RAPPAPORT, MALKA, and BETH LEVIN. 1988. What to do with $\theta$-roles. Syntax and semantics vol. 21: Thematic relations, ed. by Wendy Wilkins, 7-36. San Diego: Academic Press.

ROEPER, THOMAS. 1981. On the deductive model and the acquisition of productive morphology. In Baker \& McCarthy, 129-50.

SIEGEL, DOROTHY. 1973. Nonsources of unpassives. Syntax and Semantics vol. 2, ed. by John P. Kimball, 301-17. New York: Seminar Press.

SHIMAMURA, REIKo. 1990. Eigo no gokeisei to sono seisansei. Tokyo: Liber Press.

SMITH, CARLOTA S. 1981. Comments. In Baker \& McCarthy, 151-64.

SUGIOKA, YOKO. 1989. Haseigo ni okeru doosi sosei no uketugi. Nihongogaku no shintenkai, ed. by Susumu Kuno and Masayoshi Shibatani, 167-85. Tokyo: Kuroshio.

WOOLFORD, ELLEN. 1984. Dative verbs with unspecified objects: Optional Subcategorization versus lexical detransitivization. The Linguistic Review 3.389409 .

ZUBIZARRETA, MARIA LUISA. 1987. Levels of representation in the lexicon and in the syntax. (Studies in generative grammar, 31.) Dordrecht: Foris. 